\title{
On the strain rate dependence of the critical strain for plastic instabilities in Al-Mg alloys
}

\author{
N. Chibane ${ }^{\text {a }}$, H. Ait-Amokhtar ${ }^{a, *}$, C. Fressengeas ${ }^{\text {b }}$ \\ ${ }^{a}$ Laboratoire de Physico-Chimie des Matériaux et Catalyse (LPCMC), Faculté des Sciences Exactes, \\ Université de Bejaia, 06000 Bejaia, Algérie \\ ${ }^{\text {b }}$ Laboratoire d'Etude des Microstructures et de Mécanique des Matériaux (LEM3), \\ Université de Lorraine/CNRS, Ile du Saulcy, 57045 Metz Cedex, France
}

\begin{abstract}
The strain/strain rate range of plastic instability in $\mathrm{Al}-\mathrm{Mg}$ alloys is analyzed within the dynamic strain aging interpretations of the Portevin-Le Chatelier effect. The analysis focuses on several phenomena occurring around the minimum of the critical strain for the onset of serrations vs. applied strain rate curve. The analysis of the strain rate dependence of the flow stress strain rate sensitivity in the neighborhood of this critical point allows explaining the extension of the stability region to larger strains in the inverse behavior range and the shift to larger strain rates of the critical point when the Mg content increases.
\end{abstract}

Keywords: Aluminum alloys; Dynamic strain aging; Portevin-Le Chatelier effect; Strain rate sensitivity; Critical strain.

*Corresponding author. Tel.: +213 6621788 15; fax: +213 34215986.

E-mail address: aitamokhtar_h@yahoo.fr(H. Ait-Amokhtar). 
In Al-Mg alloys, the Portevin-Le Chatelier (PLC) effect, or jerky flow, appears around room temperature in some range of strain and strain rate. It is characterized by spatiotemporal patterns of plastic strain localization within bands. The bands are static at low applied strain rates (type C bands), they intermittently hop at medium strain rates (type B) and continuously propagate at high strain rates (type A) [1-3]. The PLC effect is due to dynamic strain aging (DSA), a phenomenon resulting from the interaction between solute atoms and mobile dislocations [1,46]. The solute atoms diffuse towards dislocations during their temporary arrests at local obstacles, and consequently increase the yield stress by additionally pinning dislocations. Such a mechanism is more efficient, and obstacle overcoming becomes more difficult, when the waiting time of dislocations at obstacles increases, i.e. when the applied strain rate decreases. The resulting "anti-thermally activated" variation of the yield stress can prevail when the characteristic time of solute diffusion becomes of the order of the loading characteristic time, in a certain strain/strain rate and temperature range. In such a case, the total strain rate sensitivity (SRS) of the flow stress may become negative, and the plastic strain may localize into narrow deformation bands reflected by serrations in the stress-strain curves at constant applied strain rate.

In the present work, we first determine the strain/strain rate domain of instability of plastic flow in the $\mathrm{Al}-2.5 \% \mathrm{Mg}$ alloy at room temperature and at imposed strain rate ranging from $10^{-6}$ to $10^{-2} \mathrm{~s}^{-1}$. The chemical composition, in weight percent, of the studied alloy is $\mathrm{Al}-2.52 \% \mathrm{Mg}-$ $022 \% \mathrm{Mn}-0.37 \% \mathrm{Cr}-0.09 \% \mathrm{Si}-0.35 \% \mathrm{Fe}-0.02 \% \mathrm{Cu}$. The samples were heat treated in air for recovery after rolling (at $400{ }^{\circ} \mathrm{C}$ for $2 \mathrm{~h}$ ) and quenched in water. In a second part, we interpret the strain rate dependence of the critical strain for the onset of serrations within the framework provided by standard interpretations of DSA. Reference is made to our previous work $[2,7,8]$ to analyze the effects of the $\mathrm{Mg}$ content on jerky flow in the neighborhood of the (doubly) critical state where the critical strain goes through its minimum. 
At room temperature and for all imposed strain rates ranging from $10^{-6}$ to $10^{-2} \mathrm{~s}^{-1}$, the plastic flow in the Al-2.5\% Mg alloy becomes unstable beyond a critical plastic strain $\varepsilon_{\mathrm{C}}$. The SRS of the flow stress was found to be negative and decreasing with strain. This trend suggests that DSA is enhanced by strain hardening. As a result, the magnitude of the stress drops increases with strain and shifts between instability types may occur, from Type A to Type B and from Type B to Type C, both transitions respectively observed at $210^{-3} \mathrm{~s}^{-1}$ and $210^{-5} \mathrm{~s}^{-1}$ in the Al$2.5 \% \mathrm{Mg}$ alloy.

Fig. 1 shows the variations of the critical plastic strain $\varepsilon_{C}$ and of the plastic strain at failure $\varepsilon_{\mathrm{r}}$ when the imposed strain rate $\dot{\varepsilon}_{a}$ increases: both decrease first, and then increase. The descending branch of the curve $\varepsilon_{C}\left(\dot{\varepsilon}_{\mathrm{a}}\right)$ is said to reflect "inverse" behavior of the critical strain (meaning that such behavior is not expected from a "normal" thermally activated mechanism). The ascending branch corresponds to "normal" behavior of $\varepsilon_{C}$. The minimum of the curve $\varepsilon_{\mathrm{C}}\left(\dot{\varepsilon}_{\mathrm{a}}\right)$, which corresponds to the switch from "inverse" to "normal" behavior, is situated around $210^{-3} \mathrm{~s}^{-1}$ in the present alloy. It correlates with a gradual shifting of PLC bands from type A to type B with increasing strain, in agreement with results previously reported in Al$\operatorname{Mg}$ alloys [2,7-9]. The plastic strain at failure $\varepsilon_{\mathrm{r}}$ first decreases, then increases when the applied strain rate increases. Hence, a reduction of the ductility of the Al-2.5\% Mg alloy occurs at room temperature in the PLC domain. The parallel evolutions of $\varepsilon_{\mathrm{C}}$ and of $\varepsilon_{\mathrm{r}}$ suggest that the reduction of the ductility is a direct consequence of the negative SRS $[1,10]$. 


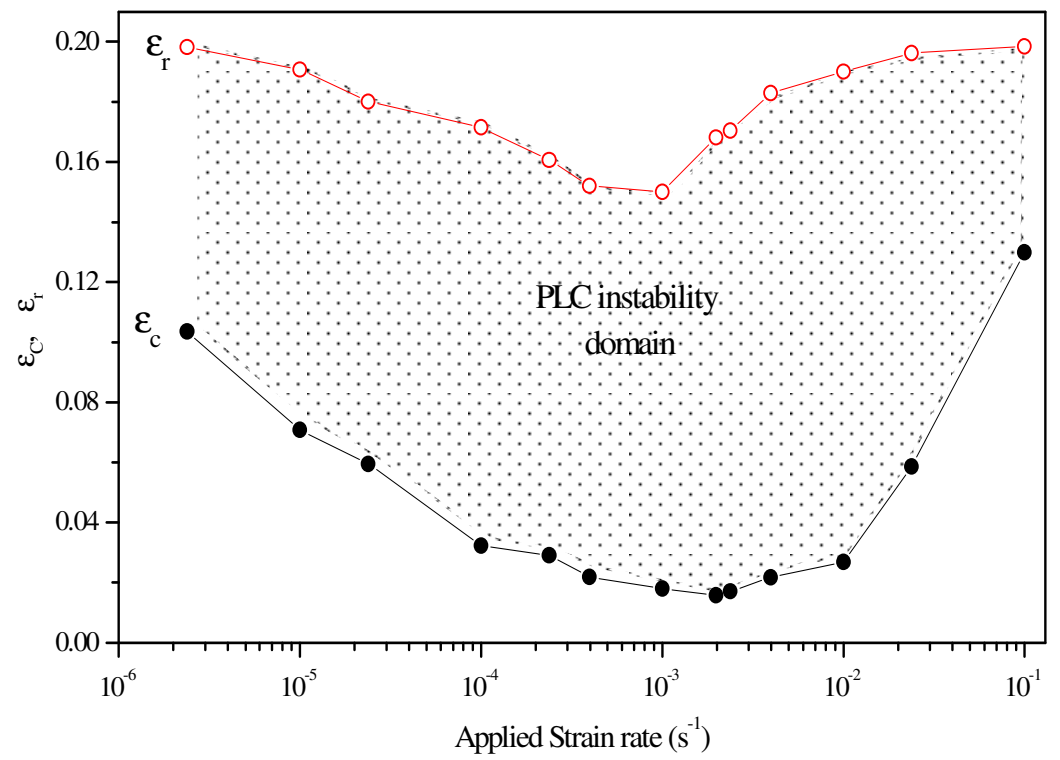

Fig.1. PLC instability domain of the Al-2.5\% Mg alloy at room temperature. Effect of the imposed strain rate on the critical plastic strain $\varepsilon_{\mathrm{c}}$ and on the plastic strain at failure $\varepsilon_{\mathrm{r}}$.

To analyze the effects of the applied strain rate $\dot{\varepsilon}_{\mathrm{a}}$ on $\varepsilon_{\mathrm{C}}$ at a prescribed temperature $\mathrm{T}$, we sketch the usual inverse-normal behavior $\varepsilon_{\mathrm{C}}\left(\dot{\varepsilon}_{\mathrm{a}}\right)$ shown in Fig. 1. According to DSA models [4,5,10-12], the uniform plastic flow becomes unstable if the SRS of the flow stress, S, becomes negative, i.e., $S=0$ is a necessary condition for instability. Due to the stabilizing influence of work hardening, instability may appear only when the SRS of the flow stress is less than a negative value of the order of $1-3 \mathrm{MPa}$ [13], a value reached in the present alloy. Thus, the curve $\varepsilon_{\mathrm{C}}\left(\dot{\varepsilon}_{\mathrm{a}}\right)$ marking the transition from stable to unstable plastic flow closely corresponds to the crossover between positive SRS and negative SRS regions in the strain/applied strain rate map. The sign of $S$ is indicated as a function of strain $\varepsilon$ and strain rate $\dot{\varepsilon}$ in Fig. 1. For a given applied strain rate, $S(\varepsilon)$ is first positive then it decreases to zero at the critical strain $\varepsilon_{C}$, and finally becomes negative in the PLC domain. For the critical applied strain rate $\dot{\varepsilon}_{\mathrm{aC}}, \varepsilon_{\mathrm{C}}$ reaches its minimum value $\varepsilon_{\mathrm{C} 0}$. At this doubly critical point, the instability is postponed to larger strains 
whether $\dot{\varepsilon}_{\mathrm{a}}$ is increased or decreased. Thus, at strain $\varepsilon_{\mathrm{C} 0}, \mathrm{~S}\left(\dot{\varepsilon}_{\mathrm{a}}\right)$ is constantly positive, except at strain rate $\dot{\varepsilon}_{\mathrm{aC}}$ where it vanishes. Below $\varepsilon_{\mathrm{C} 0}, \mathrm{~S}\left(\dot{\varepsilon}_{\mathrm{a}}\right)$ is always positive, and for strains $\varepsilon>\varepsilon_{\mathrm{C} 0}$ it becomes negative in a limited strain rate range. Thus, $\mathrm{S}\left(\dot{\varepsilon}_{\mathrm{a}}\right)$ always admits a minimum value whatever the strain. From this analysis, consistent with experimental results $[2,7,9,14,15]$, we investigate hereafter the SRS and the necessary condition for instability.

The constitutive law providing the strain $\varepsilon$ and strain rate $\dot{\varepsilon}$ dependences of the flow stress can be expressed as follows [10]:

$$
\sigma(\varepsilon, \dot{\varepsilon})=\mathrm{h} \varepsilon+\mathrm{S}_{0} \ln \left(\dot{\varepsilon} / \dot{\varepsilon}^{*}\right)+\mathrm{F}(\dot{\varepsilon})
$$

Here, $\mathrm{h}$ is the work-hardening coefficient and $\mathrm{S}_{0} \ln \left(\dot{\varepsilon} / \dot{\varepsilon}^{*}\right)$ reflects the "normal" thermal activation mechanism for dislocation breakaway. $\dot{\varepsilon}^{*}$ is a reference strain rate and $\mathrm{S}_{0}$ is the SRS of the flow stress in the absence of DSA. For the sake of simplicity, $\mathrm{S}_{0}$ will be considered as a constant. $\mathrm{F}(\dot{\varepsilon})$ is the additional pinning stress due to strain aging. Following Louat [16], it can be given by the expression:

$$
\mathrm{F}(\dot{\varepsilon})=\mathrm{f}_{0}\left(1-\exp -(\Omega / \tau \dot{\varepsilon})^{\mathrm{n}}\right)
$$

where:

$\Omega$ is the elementary incremental strain obtained when all mobile dislocations accomplish a successful activation event [17]. $\Omega$ is related to the mobile and forest dislocation densities, $\rho_{\mathrm{m}}$ and $\rho_{\mathrm{f}}$ respectively, by the expression:

$$
\Omega=b \rho_{\mathrm{m}} \rho_{\mathrm{f}}^{-1 / 2}
$$

where $b$ is the Burgers vector modulus. If $t_{w}$ is the waiting time of dislocations on their obstacles and $\Delta \mathrm{l}=\rho_{\mathrm{f}}^{-1 / 2}$ the mean dislocation free path, the Orowan relation provides the relationship 


$$
\dot{\varepsilon}=\rho_{\mathrm{m}} \mathrm{bv} \cong \rho_{\mathrm{m}} \mathrm{b}\left(\Delta \mathrm{l} / \mathrm{t}_{\mathrm{w}}\right)=\rho_{\mathrm{m}} \mathrm{b}\left(\rho_{\mathrm{f}}^{-1 / 2} / \mathrm{t}_{\mathrm{w}}\right)=\Omega / \mathrm{t}_{\mathrm{w}}
$$

As $\rho_{\mathrm{m}}$ and $\rho_{\mathrm{f}}$ are strain and strain rate dependent, $\Omega$ also depends on these quantities: therefore, we may write $\Omega=\Omega(\varepsilon, \dot{\varepsilon})$.

$\tau$ is the characteristic time associated with solute diffusion, which depends on the binding energy between solute atoms and dislocations, on the diffusion coefficient of solute atoms and on solute concentration.

$\mathrm{f}_{0}$ represents the pinning stress at saturation. It is directly proportional to the solute concentration of the element responsible for DSA, i.e. Mg atoms in Al-Mg alloys [18].

$\mathrm{n}$ is an exponent reflecting the type of solute diffusion mechanism associated with DSA. It is equal to $2 / 3$ and $1 / 3$ respectively for bulk diffusion and pipe diffusion.

From Eqs.(1) and (2), we obtain the total SRS of the flow stress:

$$
S=\frac{\partial \sigma}{\partial \ln \dot{\varepsilon}}=\mathrm{S}_{0}-\mathrm{nf}_{0} \mathrm{X}(\varepsilon, \dot{\varepsilon}) \exp (-\mathrm{X}(\varepsilon, \dot{\varepsilon}))\left(1-\frac{\partial \Omega(\varepsilon, \dot{\varepsilon})}{\partial \dot{\varepsilon}} \cdot \frac{\dot{\varepsilon}}{\Omega(\varepsilon, \dot{\varepsilon})}\right)
$$

where $\mathrm{X}=(\Omega(\varepsilon, \dot{\varepsilon}) / \tau \dot{\varepsilon})^{\mathrm{n}}$.

Provisionally considering $\Omega$ to be independent of $\dot{\varepsilon}, \Omega(\varepsilon, \dot{\varepsilon})=\Omega(\varepsilon)$, we find the expression of the SRS, noted $\mathrm{S}_{\mathrm{KE}}$ in the following, obtained in the Kubin-Estrin (KE) model [17]:

$$
\mathrm{S}_{\mathrm{KE}}=\mathrm{S}_{0}+\mathrm{S}_{\mathrm{DSA}}
$$

$\mathrm{S}_{\mathrm{KE}}$ is expressed as the sum of a positive term $\mathrm{S}_{0}$, obtained from normal thermal activation of dislocation obstacle overcoming, and a negative term, $\mathrm{S}_{\mathrm{DSA}}$, which is added in the presence of DSA. Thus, Eq.(5) can be rewritten as follows:

$$
\mathrm{S}=\mathrm{S}_{0}+\mathrm{S}_{\mathrm{DSA}}[1+\delta(\varepsilon, \dot{\varepsilon})]
$$

where

$$
\delta(\varepsilon, \dot{\varepsilon})=-\frac{\partial \Omega(\varepsilon, \dot{\varepsilon})}{\partial \dot{\varepsilon}} \frac{\dot{\varepsilon}}{\Omega(\varepsilon, \dot{\varepsilon})}=-\frac{\partial \ln \Omega}{\partial \ln \dot{\varepsilon}}
$$


Therefore, the SRS can be expressed as a sum of three contributions:

$$
\mathrm{S}=\mathrm{S}_{0}+\mathrm{S}_{\mathrm{DSA}}+\mathrm{S}_{\dot{\varepsilon}}
$$

where $\mathrm{S}_{\dot{\varepsilon}}=\mathrm{S}_{\text {DSA }} \delta$ is a negative additional component due to the strain rate sensitivity of $\Omega$. In the present work, the effects of the strain rate on $\Omega$ are modeled using the KE model [17] for the dislocation density dynamics. The strain dependence of the mobile and forest dislocations densities are described by the following set of coupled differential equations:

$$
\left\{\begin{array}{l}
\frac{\partial \rho_{\mathrm{m}}}{\partial \varepsilon}=\frac{\mathrm{C}_{1}}{\mathrm{~b}^{2}}-\mathrm{C}_{2} \rho_{\mathrm{m}}-\frac{\mathrm{C}_{3}}{\mathrm{~b}} \rho_{\mathrm{f}}^{1 / 2} \\
\frac{\partial \rho_{\mathrm{f}}}{\partial \varepsilon}=\mathrm{C}_{2} \rho_{\mathrm{m}}+\frac{\mathrm{C}_{3}}{\mathrm{~b}} \rho_{\mathrm{f}}^{1 / 2}-\mathrm{C}_{4} \rho_{\mathrm{f}}
\end{array}\right.
$$

where the parameters $C_{i}$ are related to the multiplication of mobile dislocations $\left(C_{1}\right)$, their mutual annihilation and trapping $\left(\mathrm{C}_{2}\right)$, their immobilization through interaction with forest dislocations $\left(\mathrm{C}_{3}\right)$ and to dynamic recovery $\left(\mathrm{C}_{4}\right)$. To account for the effects of the strain rate on $\Omega$, we assume that an increase in $\dot{\varepsilon}$ reduces dynamic recovery $[17,19]$, which induces a decrease of the parameters $\mathrm{C}_{2}$ and $\mathrm{C}_{4}$ with increasing strain rate, and further that $\mathrm{C}_{2}$ is less sensitive to strain rate [19]. $\mathrm{C}_{2}$ and $\mathrm{C}_{4}$ are therefore expressed as follows:

$$
\left\{\begin{array}{l}
\mathrm{C}_{2}=\mathrm{C}_{2_{0}}\left(\frac{\dot{\varepsilon}}{\dot{\varepsilon}_{0}}\right)^{-\mathrm{m}_{2}} \\
\mathrm{C}_{4}=\mathrm{C}_{4_{0}}\left(\frac{\dot{\varepsilon}}{\dot{\varepsilon}_{0}}\right)^{-\mathrm{m}_{4}}
\end{array}\right.
$$

where the exponents $\mathrm{m}_{2}$ and $\mathrm{m}_{4}$ are constants $\left(\mathrm{m}_{2}<<\mathrm{m}_{4}\right), \dot{\varepsilon}_{0}$ is a constant strain rate and $\mathrm{C}_{2_{0}}$ and $\mathrm{C}_{4_{0}}$ are the values of the parameters when the effect of strain rate is not accounted for. We use the values $\dot{\varepsilon}_{0}=510^{-4} \mathrm{~s}^{-1}, \mathrm{~m}_{2}=0.02$ and $\mathrm{m}_{4}=0.2$. The values chosen for the parameters involved in Eqs. (10) and (11) are reported in Table 1. $\rho_{\mathrm{m} 0}$ and $\rho_{\mathrm{f} 0}$ are the initial dislocation densities, $\rho_{\mathrm{ms}}$ and $\rho_{\mathrm{fs}}$ are their values at saturation. 


\begin{tabular}{ccccccccc}
\hline$\rho_{m 0}\left(m^{-2}\right)$ & $\rho_{f 0}\left(m^{-2}\right)$ & $\rho_{m s}\left(m^{-2}\right)$ & $\rho_{f s}\left(m^{-2}\right)$ & $b(m)$ & $C_{1}$ & $C_{20}$ & $C_{3}$ & $C_{40}$ \\
\hline $10^{10}$ & $10^{11}$ & $510^{13}$ & $10^{14}$ & $310^{-10}$ & $310^{-5}$ & 0.606 & 0.009 & 3.333 \\
\hline
\end{tabular}

Table 1: Values of the parameters chosen to solve numerically Eqs. (10-a) and (10-b).

Fig. 2 shows the strain dependence of the elementary strain $\Omega$ at different strain rates, obtained from the numerical solution of the set of equations $(10-a)$ and $(10-b)$. It shows that $\Omega$ is a decreasing function of strain rate. It increases monotonously with strain at low strain rate, but exhibits a maximum and then decreases with strain at high strain rate. The effect of the strain rate on $\Omega$ is clearly shown in Fig. 3 through its first derivative with respect to strain rate at different strains. $\Omega$ depends strongly on strain rate at low strain rates, but very weakly at high strain rates.

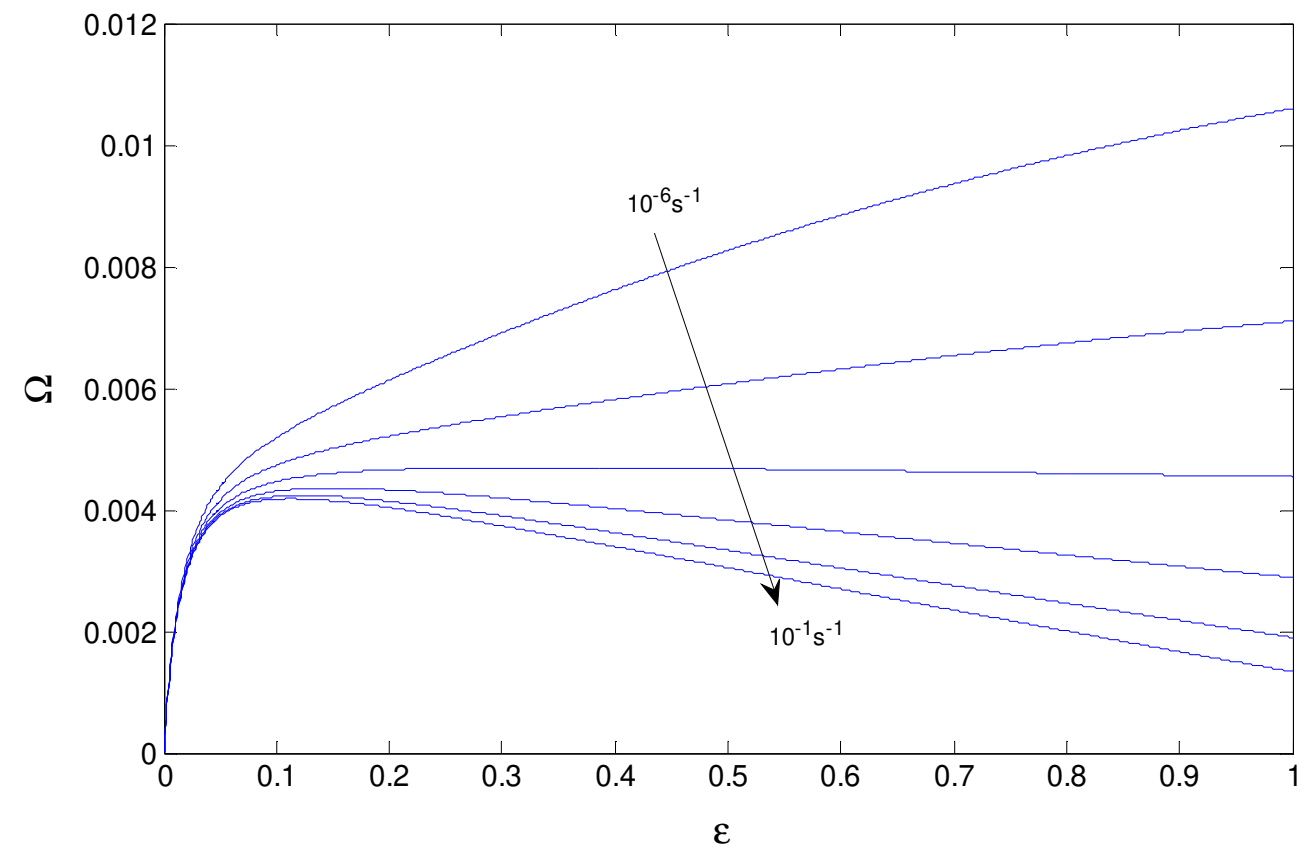

Fig.2. Strain dependence of the elementary strain $\Omega$ at strain rates $10^{-6}, 10^{-5}, 10^{-4}, 10^{-3}, 10^{-2}$ and $10^{-1} \mathrm{~s}^{-1}$. 


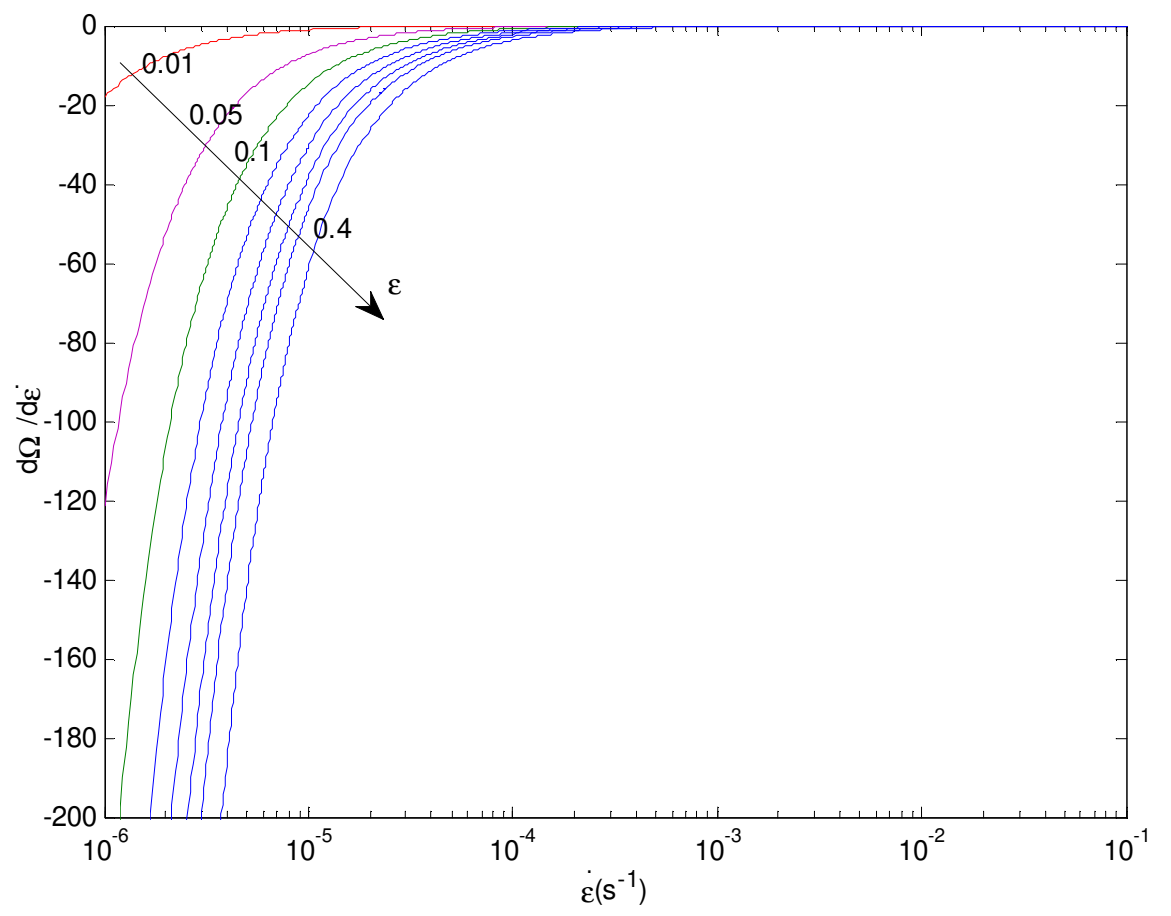

Fig.3. Effect of strain rate on the first derivative of $\Omega$ at different strains.

We now analyze the strain rate sensitivity of the SRS around the critical point $\mathrm{C}\left(\dot{\varepsilon}_{\mathrm{aC}}, \varepsilon_{\mathrm{C} 0}\right)$ showed in Fig. 1. From Eq.(5), the variation of the SRS with strain rate is

$$
\begin{aligned}
\left.\frac{\partial S}{\partial \dot{\varepsilon}}\right)= & n^{2} f_{0} X \exp (-X) \frac{1}{\dot{\varepsilon}}[1-X] \\
& -n f_{0} X \exp (-X) \frac{1}{\Omega^{2}}\left[\frac{\partial \Omega}{\partial \dot{\varepsilon}} \Omega(2 n-2 X n-1)+\left(\frac{\partial \Omega}{\partial \dot{\varepsilon}}\right)^{2} \dot{\varepsilon}(X n+1-n)-\frac{\partial^{2} \Omega}{\partial \dot{\varepsilon}^{2}} \Omega \dot{\varepsilon}\right]
\end{aligned}
$$

As the critical point $\mathrm{C}$ is located in the early stages of plastic flow, dislocation annihilation and dynamic recovery are not yet significant. Also, the applied strain rate is in the medium range, and $\Omega$ is therefore almost independent of strain rate, as shown in Fig.3. Thus, we may consider as a first step that $\Omega(\varepsilon, \dot{\varepsilon})=\Omega(\varepsilon)$ in a close vicinity of the critical point. Eq.(12) then becomes simply

$$
\left.\frac{\partial \mathrm{S}}{\partial \dot{\varepsilon}}\right)=\mathrm{n}^{2} \mathrm{f}_{0} \mathrm{X} \exp (-\mathrm{X}) \frac{1}{\dot{\varepsilon}}[1-\mathrm{X}]
$$


Eq.(13) shows that $S$ has its minimum value for $X=1$, i.e.

$$
\left(\frac{\Omega(\varepsilon)}{\tau \dot{\varepsilon}}\right)^{\mathrm{n}}=1
$$

which corresponds in particular to the critical point $\mathrm{C}\left(\dot{\varepsilon}_{\mathrm{aC}}, \varepsilon_{\mathrm{C} 0}\right)$, where

$$
\dot{\varepsilon}_{\mathrm{aC}}=\frac{\Omega\left(\varepsilon_{\mathrm{C} 0}\right)}{\tau}
$$

The characteristic time for solute diffusivity, $\tau$, may be determined from this relation. Considerer two values for the elementary strain $\Omega, 2 \times 10^{-3}$ and $4 \times 10^{-3}$, which are reasonable estimates at low plastic strains [17,20]. $\dot{\varepsilon}_{\mathrm{aC}}$ is experimentally determined from the curve $\varepsilon_{\mathrm{C}}\left(\dot{\varepsilon}_{\mathrm{a}}\right)$ (Fig.1); considering values reported earlier in $\mathrm{Al}-1 \% \mathrm{Mg}, \mathrm{Al}-2 \% \mathrm{Mg}, \mathrm{Al}-3.2 \% \mathrm{Mg}$ and Al-4.5\% Mg alloys [7], we obtain Fig. 4 where the effect of the nominal concentration of $\mathrm{Mg}$ on $\tau$ is shown. It is seen that solute diffusivity is faster, and hence more effective, when the solute concentration is increased.

As shown by the semi-empirical study of Horváth at al. [20] in Al-Mg alloys, $\Omega$ increases with the Mg content. Since $\tau$ decreases with the Mg content, Eq.(15) shows that the critical strain rate $\dot{\varepsilon}_{\mathrm{aC}}$ shifts to larger values when the Mg content increases. This result agrees with our earlier experimental reports in Al-Mg alloys [7], where it is found that $\dot{\varepsilon}_{\mathrm{aC}}$ shifts from $310^{-4} \mathrm{~s}^{-1}$ to 4 $10^{-3} \mathrm{~s}^{-1}$ when the $\mathrm{Mg}$ content increases from 1 to $4.5 \% \mathrm{Mg}$.

As indicated above, experimental reports on the strain rate dependence of the critical strain for plastic instability in Al-Mg alloys show that, around the minimum of the critical strain vs. applied strain rate curve, $\mathrm{C}\left(\dot{\varepsilon}_{\mathrm{aC}}, \varepsilon_{\mathrm{C} 0}\right)$, several changes with strain and strain rate occur in the unstable behavior: the evolution of the critical strain switches from "inverse" to "normal", the type of instability changes from type A to type B with increasing strain, and from type B to type A with increasing strain rate. As Eqs. $(5,15)$ show, $\dot{\varepsilon}_{\mathrm{aC}}$ is an equilibrium strain rate where the 


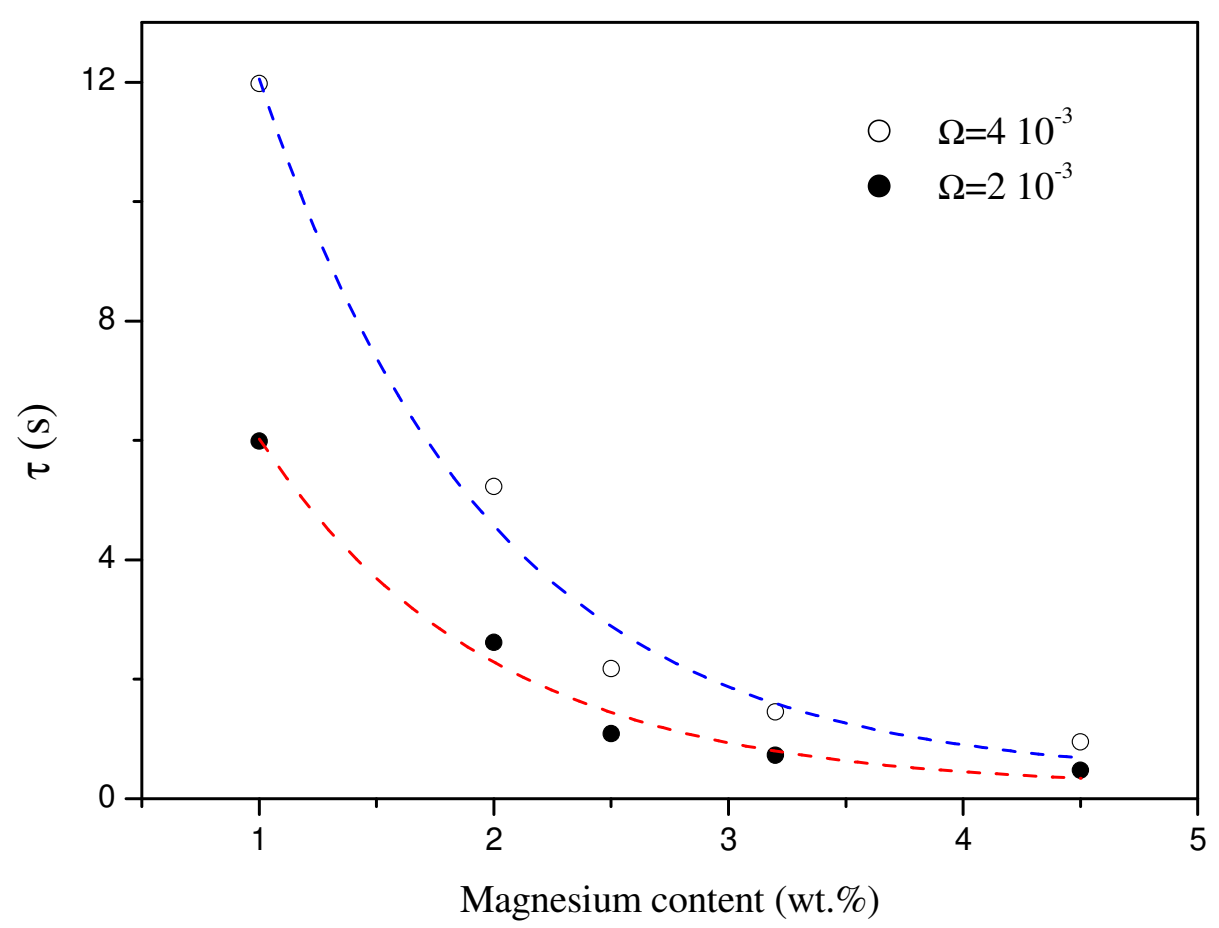

Fig.4. Effect of the nominal concentration of $\mathrm{Mg}$ on $\tau$, the characteristic time for solute diffusivity.

characteristic time $\tau$ for solute diffusivity is exactly the waiting time $\mathrm{t}_{\mathrm{w}}$ of dislocations at the obstacles controlling plasticity. Two regimes can therefore be defined on either sides of $\dot{\varepsilon}_{\mathrm{aC}}$. At high applied strain rates, $\mathrm{t}_{\mathrm{w}}<<\tau$, diffusivity is less effective and the instability is controlled by plasticity. In this range, we observed in a previous report that the $\mathrm{Mg}$ concentration weakly affects the critical strain [7]. At low applied strain rates, $t_{w}>>$, diffusivity is more effective and it becomes the controlling mechanism of instability. Our observations showed that the critical strain then becomes strongly dependent on the $\mathrm{Mg}$ content [7]. Indeed, as Fig.4 allows to predict, diffusivity and consequently DSA become more and more effective when the Mg content increases, which induces critical strain reduction. 
Further, around the minimum of the curve $\varepsilon_{\mathrm{C}}\left(\dot{\varepsilon}_{\mathrm{a}}\right)$, which can be locally approximated by a parabola, Eqs. $(12,13)$ show that the first derivative of $\mathrm{S}$ with respect to strain rate is proportional to $\mathrm{f}_{0}$, itself being proportional to the $\mathrm{Mg}$ concentration [18]. Thus, decreasing the $\mathrm{Mg}$ content amounts to reducing the curvature of the curve $\varepsilon_{C}\left(\dot{\varepsilon}_{\mathrm{a}}\right)$ and, consequently, to enlarging in strain the PLC domain. Note that the additional SRS due to the strain rate dependence of $\Omega$, i.e. the term $S_{\dot{\varepsilon}}$ in Eq.(9), further lowers the critical strain at low strain rates but not at high strain rates where it can be neglected. Hence, the strain rate dependence of $\Omega$ contributes to explaining why the PLC domain is more enlarged in the inverse behavior sub-domain than in the normal behavior sub-domain.

In summary, we determined the strain/strain rate domain of instability of plastic flow in the Al$2.5 \% \mathrm{Mg}$ alloy at room temperature and we analyzed, using the DSA interpretation of the PLC effect, the plastic flow around the critical strain rate (where the critical strain for the onset of serrations reaches its minimum). The study allows determination of the characteristic solute diffusion time. The latter is equal, at the corresponding critical strain rate, to the waiting time of dislocations at local obstacles. We also explain the extension of the stability region to larger strains at low strain rates and the shift to larger values of the critical strain rate when the $\mathrm{Mg}$ content increases.

\section{Acknowledgements}

H. A.-A. is thankful to University of Bejaia (Algeria) for financial support and the people in charge of LEM3 (University of Lorraine, France) for their welcome and help.

\section{References}

[1] L.P. Kubin, Y. Estrin, J. Phys. III 1 (1991) 929-943.

[2] H. Ait-Amokhtar, S. Boudrahem, C. Fressengeas, Scripta Mater. 54 (2006) 2113-2118. 
[3] K. Chihab, Y. Estrin, L.P. Kubin, J. Vergnol, Scripta Metall. 21 (1987) 203-208.

[4] P.G. McCormick, Acta Metall. 20 (1972) 351-354.

[5] A. Van den Beukel, Phys. Status Solidi (a) 30 (1975) 197-206.

[6] H. Aboulfadl, J. Deges, P. Choi, D. Raabe, Acta Mater. 86 (2015) 34-42.

[7] H. Ait-Amokhtar, C. Fressengeas, K. Bouabdallah, Mater Sci Eng A 631(2015) 209-213.

[8] H. Ait-Amokhtar, C. Fressengeas, Acta Mater. 58 (2010) 1342-1349.

[9] R. Král, P. Lukáč, Mater. Sci. Eng. A 234-236 (1997) 786-789.

[10] Y. Estrin and L.P. Kubin, Spatial coupling and propagative plastic instabilities. In: Muhlhaus, H.B. (Ed.), Continuum Models for Materials with Microstructures, Wiley, New York, 1995, pp. 395-450.

[11] J. Balík, P. Lukác, L.P. Kubin, Scripta Mater. 42 (2000) 465-471.

[12] P. Penning, Acta Metall. 20 (1972) 1169-1175.

[13] C. Fressengeas, A.J. Beaudoin, M. Lebyodkin, L.P. Kubin, Y. Estrin, Mat. Sci. Eng. A 400-401(2005) 226-230.

[14] H. Ait-Amokhtar, P. Vacher, S. Boudrahem, Acta Mater. 54 (2006) 4365-4371.

[15] S. Kumar, Scripta Metall.Mater. 33 (1995) 81-86.

[16] N. Louat, Scripta Metall. 15 (1981) 1167-1170.

[17] L.P. Kubin, Y. Estrin, Acta Metall. Mater. 38 (1990) 697-708.

[18] E. Pink, A. Grinberg, Acta Metall. 30 (1982) 2153-2160.

[19] Y. Estrin, L.P. Kubin, Acta Metall. 34 (1986) 24552464.

[20] G. Horváth, N. Q. Chinh, J. Gubicza, J. Lendvai, Mater. Sci. Eng. A 445-446 (2007) 186192. 